\title{
Supercharged eGFP-TRAIL Decorated NETs to Ensnare and Kill Disseminated Tumor Cells
}

\author{
Thong M. CaO and Michael R. King \\ Department of Biomedical Engineering, Vanderbilt University, Nashville, TN 37235, USA
}

(Received 3 May 2020; accepted 20 July 2020; published online 6 August 2020)

\begin{abstract}
Background-NETosis is an innate immune response elicited by activated neutrophils to fight microbial infections. Activated neutrophils release DNA fibers decorated with antimicrobial proteins called neutrophil extracellular traps (NETs) into the extracellular space to trap and kill surrounding microbes.

Methods-Here, we show that tumor-derived IL-8 released by cancer cells also activates the release of NETs. Until now, there have been no existing technologies that leverage NETs as an anti-tumor drug delivery vehicle. In this study, we demonstrate the re-engineering of neutrophils to express an apoptosis-inducing chimeric protein, supercharged eGFPTRAIL, on NETs that can ensnare and kill tumor cells while retaining their anti-microbial capabilities.

Results - We observed significant TRAIL-induced apoptosis in tumor cells captured by TRAIL-decorated NETs.

Conclusions - This work demonstrates NETs as a promising technology to deliver protein in response to local cytokine signals.
\end{abstract}

Keywords-NETosis, Neutrophil, Metastasis, TRAIL, Apoptosis, Protein engineering.

\section{INTRODUCTION}

Neutrophils play a significant role in all stages of tumorigenesis, from the initial genotoxic insult, to metastasis to distant organs. Chronic inflammation drives neutrophils to release mutagenetic agents, including reactive oxygen species (ROS) and hypochlorous acid $(\mathrm{HOCl})$ that induce DNA damage and mutagenicity on surrounding cells. ${ }^{2,7,9}$ Neutrophils comprise a significant percentage of white blood cells that infiltrate the tumor microenvironment. ${ }^{8}$ In that setting, infiltrating neutrophils continue to promote

Address correspondence to Michael R. King, Department of Biomedical Engineering, Vanderbilt University, Nashville, TN 37235, USA. Electronic mail: mike.king@vanderbilt.edu tumor development by secreting pro-inflammatory and pro-angiogenesis chemokines and cytokines such as matrix metallopeptidase 9 (MMP9) and interleukin 6 (IL-6). ${ }^{1,15,19}$ Tumor metastasis via hematogenous dissemination involves circulating tumor cells (CTCs) shedding from the primary tumor site and reaching distant organs through the circulatory system. In a recent study, neutrophils have been found to support CTC survival during hematogenous dissemination. ${ }^{18}$ Furthermore, neutrophils have been identified as the main driver in establishing the pre-metastatic microenvironment in several mouse breast cancer models. $^{20}$

NETosis is a unique form of innate immune response elicited primarily by neutrophils to combat microbial infections. ${ }^{3}$ In the presence of antigens, neutrophils undergo NETosis by following a program of cell death and releasing condensed DNA fibers decorated with cationic antimicrobial proteins, collectively called NETs, into the extracellular space. ${ }^{16} \mathrm{Tu}-$ mor cells release tumor-derived interleukin 8 (IL-8), a potent neutrophil chemoattractant, to increase tumor growth and metastatic potential by: (i) promoting tumor neovascularization, and (ii) inducing infiltrating neutrophils to release pro-metastatic enzymes. ${ }^{4}$

\section{RESULTS}

Initially, NETosis was found to specifically occur in the presence of bacterial antigens, but here we show that tumor-derived IL-8 released by tumor cells also elicits NETosis in neutrophils (Figs. $1 \mathrm{~b}$ and 1c). Blocking the tumor-derived IL-8 pathway with a small molecule, reparixin, returned NETosis back to basal levels (Fig. 1e). Moreover, we quantified cell-free serum DNA levels in cancer patients and found a significant increase in serum DNA level compared to the healthy cohort, suggesting that NETs may also have an elevated presence in the circulatory system in cancer 
(a)

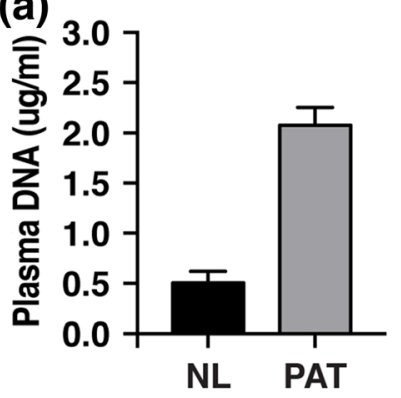

(b)
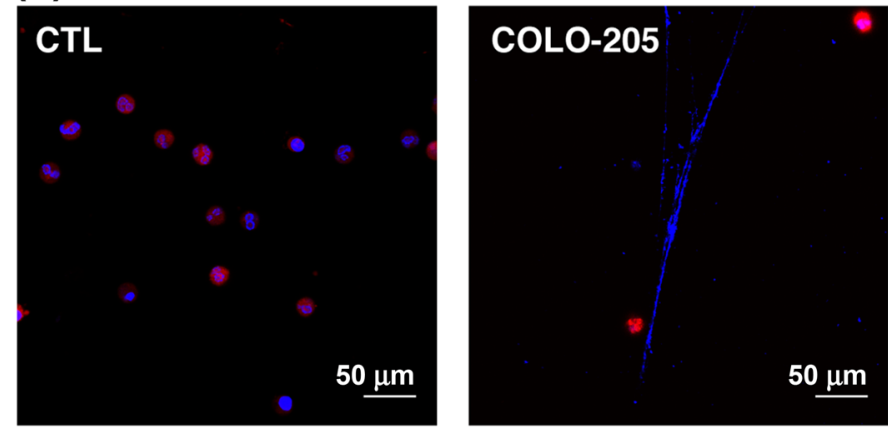

(d)
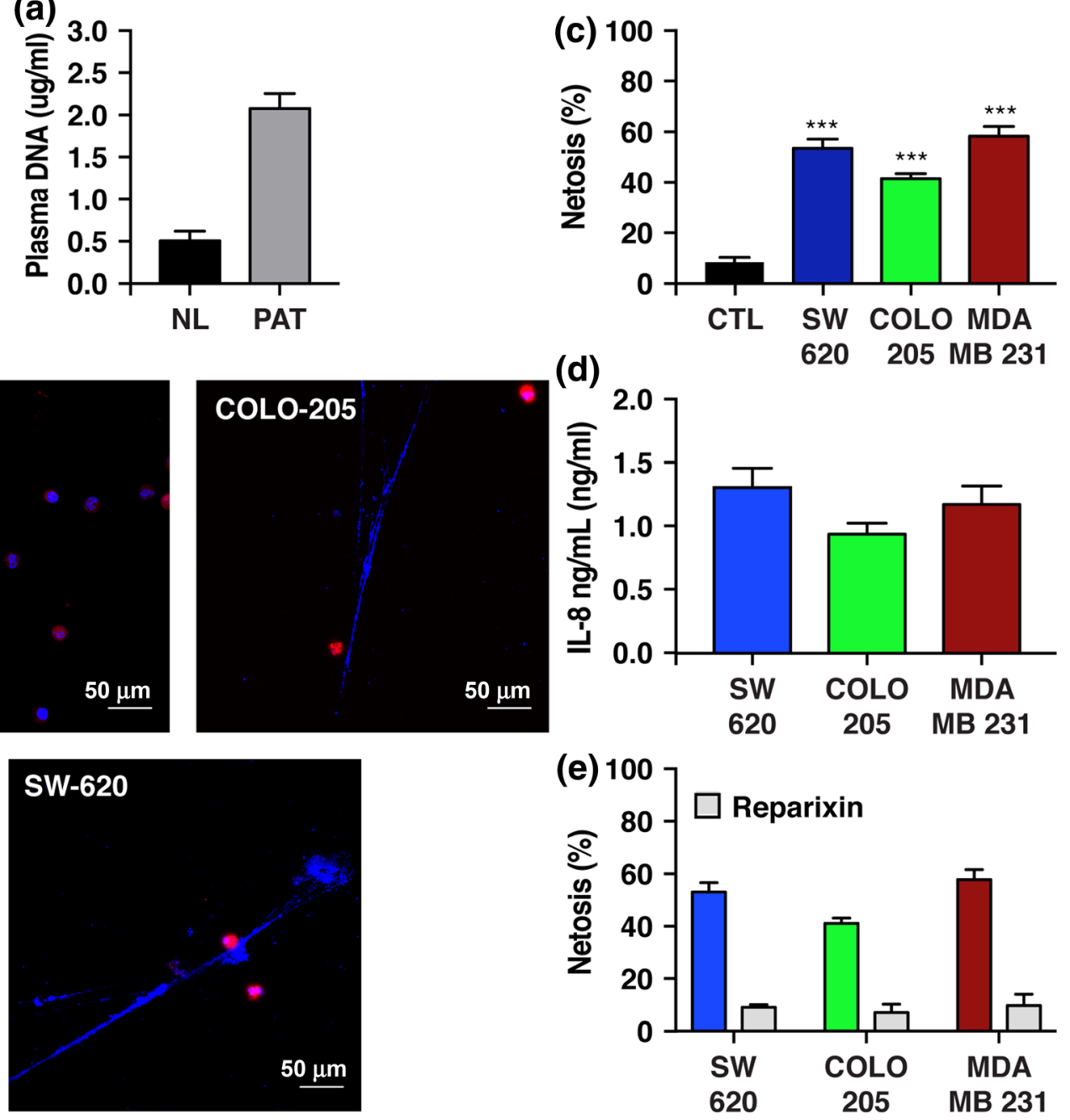

FIGURE 1. Neutrophils undergo spontaneous NETosis in the presence of tumor cells. (a) cfDNA plasma DNA level of normal donors (ND) and cancer patients (PAT). (b) Confocal images of neutrophils that underwent NETosis in conditioned media. (CTL) - control group, COLO-205 and SW-620 are conditioned media collected after $48 \mathrm{~h}$ from these indicated cell lines. (c) NETosis level of neutrophils after culturing in conditioned media for $24 \mathrm{~h}$. (d, e) Extracellular tumor-derived IL-8 levels in conditioned media. Reparixin concentration was $20 \mu \mathrm{g} / \mathrm{mL}$.

patients (Fig. 1a). These results support previous findings that malignant and non-malignant neutrophils in tumor-bearing mice have increased sensitivity toward undergoing NETosis. ${ }^{5}$ Given data shown here of the ubiquitous presence of neutrophils in all stages of tumor development, we proposed a unique anti-tumor drug delivery system by exploiting the ability of neutrophils to spontaneously undergo NETosis in the presence of cancer cells.

Although NETs have been shown to ensnare cancer cells, they lack the ability to kill cancer cells. More insidiously, trapped tumor cells show increased metastatic potential. ${ }^{6}$ In the current study, we proposed to re-engineer human neutrophils to express NETs decorated with an apoptosis-inducing peptide, TRAIL, that could selectively destroy cancer cells during NETosis. TRAIL is a small cytokine expressed by most cell types that selectively induces apoptosis in tumor cells overexpressing death receptors while sparing healthy cells. ${ }^{11,14}$ In this model, we successfully knocked in our gene of interest (GOI), expressing the chimeric protein eGFP-TRAIL, into the safe harbor site AAVS1 on chromosome 19 of the proto-neutrophilic cell line PLB-985 using the CRISPR/Cas system (Figs. 2a and 2b). PLB-985 is a leukemic cell line that can be induced into a neutrophil-like state capable of undergoing NETosis. ${ }^{12,17}$ Aside from serving as a fluorescent marker, eGFP serves a more important function by selectively allowing the chimeric protein to electrostatically bind to the DNA fibers of NETs during NETosis (Fig. 2a). By modifying the surface charge of eGFP to become increasingly more positive, we were able to increase its avidity to the negatively-charged DNA fibers in a charge-dependent 
(a)

Chromosome 19
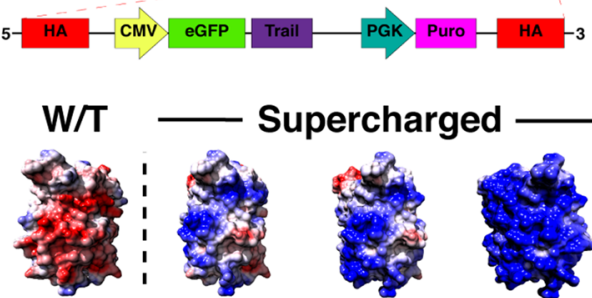

$-8$

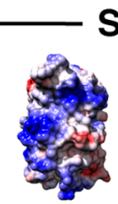

$+4$

Surface Charge
AAVS1

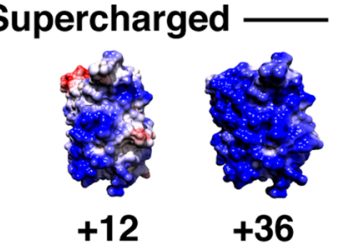

(b) 5 ' Integration

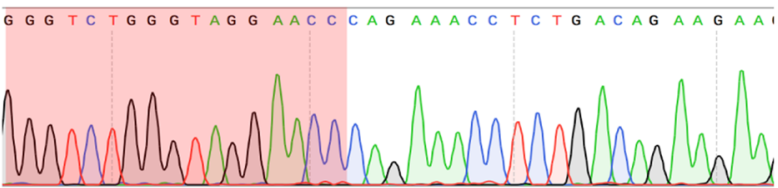

3' Integration
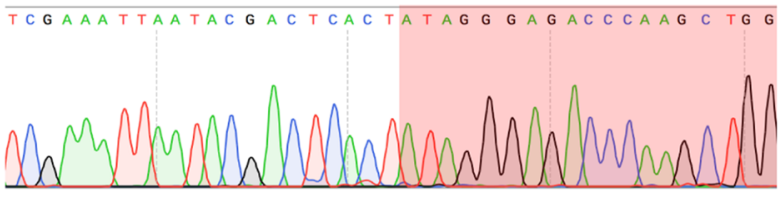

(e)
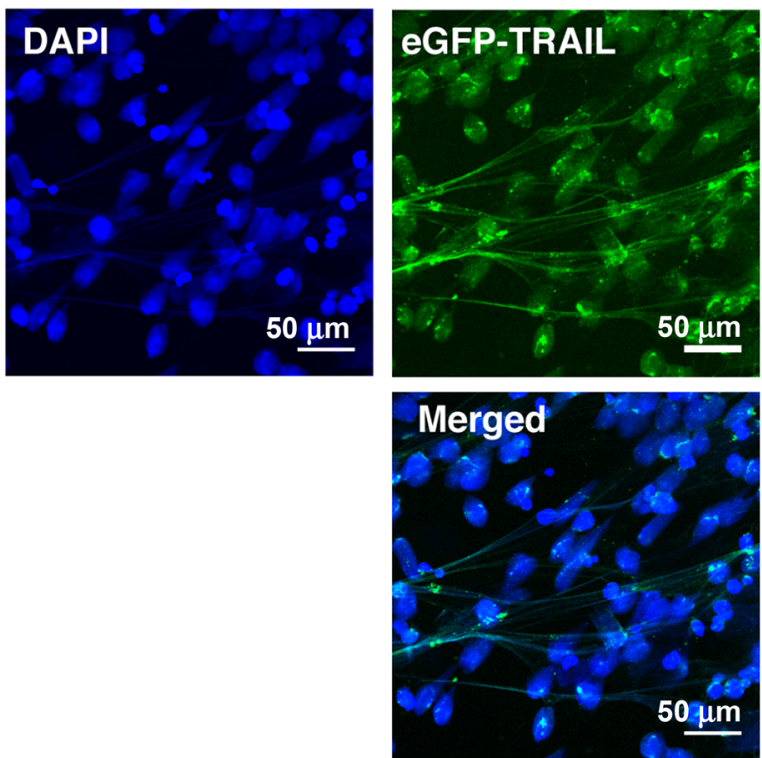

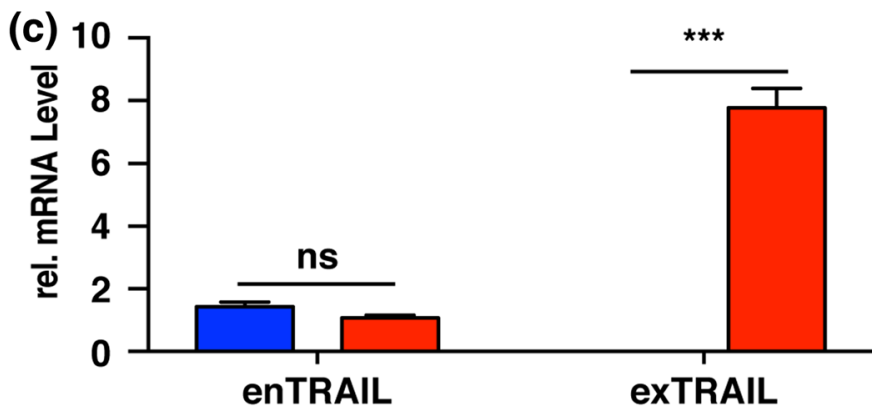

(d)

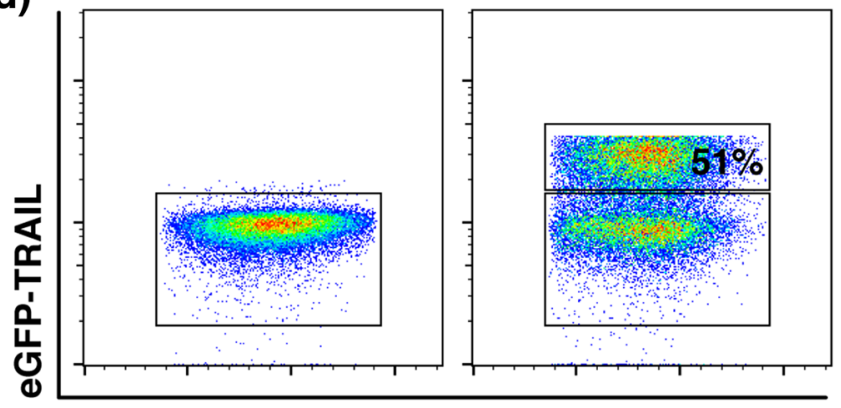

Forward Scatter
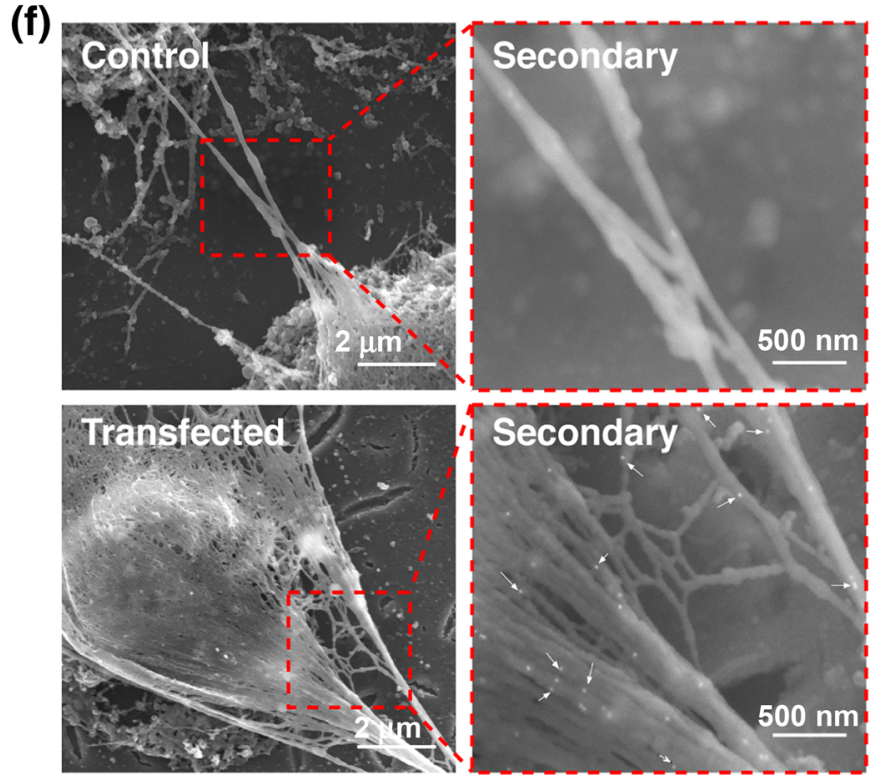

FIGURE 2. Engineered neutrophils express supercharged eGFP-TRAIL on NETs during NETosis. (a) Schematic of the insertion site, AAVS1, on chromosome 19. Cartoon representation of eGFP-TRAIL chimeric protein. Surface charge of eGFP ranging from -4 to +36 (Red-negative charge, Blue-positive charge. (b) DNA sequencing result of genomic DNA isolated from cells positive for eGFP-TRAIL. (c) relative mRNA level of endogenous (enTRAIL) and exogenous (exTRAIL) TRAIL levels. (d) Flow cytometry of cells expressing eGFP-TRAIL $24 \mathrm{~h}$ after nucleofection. (e) Confocal images of NETs decorated with eGFP-TRAIL (Blue-DAPI stain, Green-eGFP-TRAIL). (f) Immuno-gold SEM images of neutrophils expressing eGFP-decorated NETs (White arrows-eGFPTRAIL).

manner (Fig. 3). The transfected cells stably expressed eGFP-TRAIL-decorated NETs during NETosis (Figs. 2e-2f). mRNA data showed successful expression of the transgene. Immunostaining scanning elec- tron microscopy with TRAIL antibody-conjugated gold nanoparticles of NET DNA fibers revealed that eGFP-TRAIL protein molecules are decorated along the DNA fibers (Fig. 2f). 

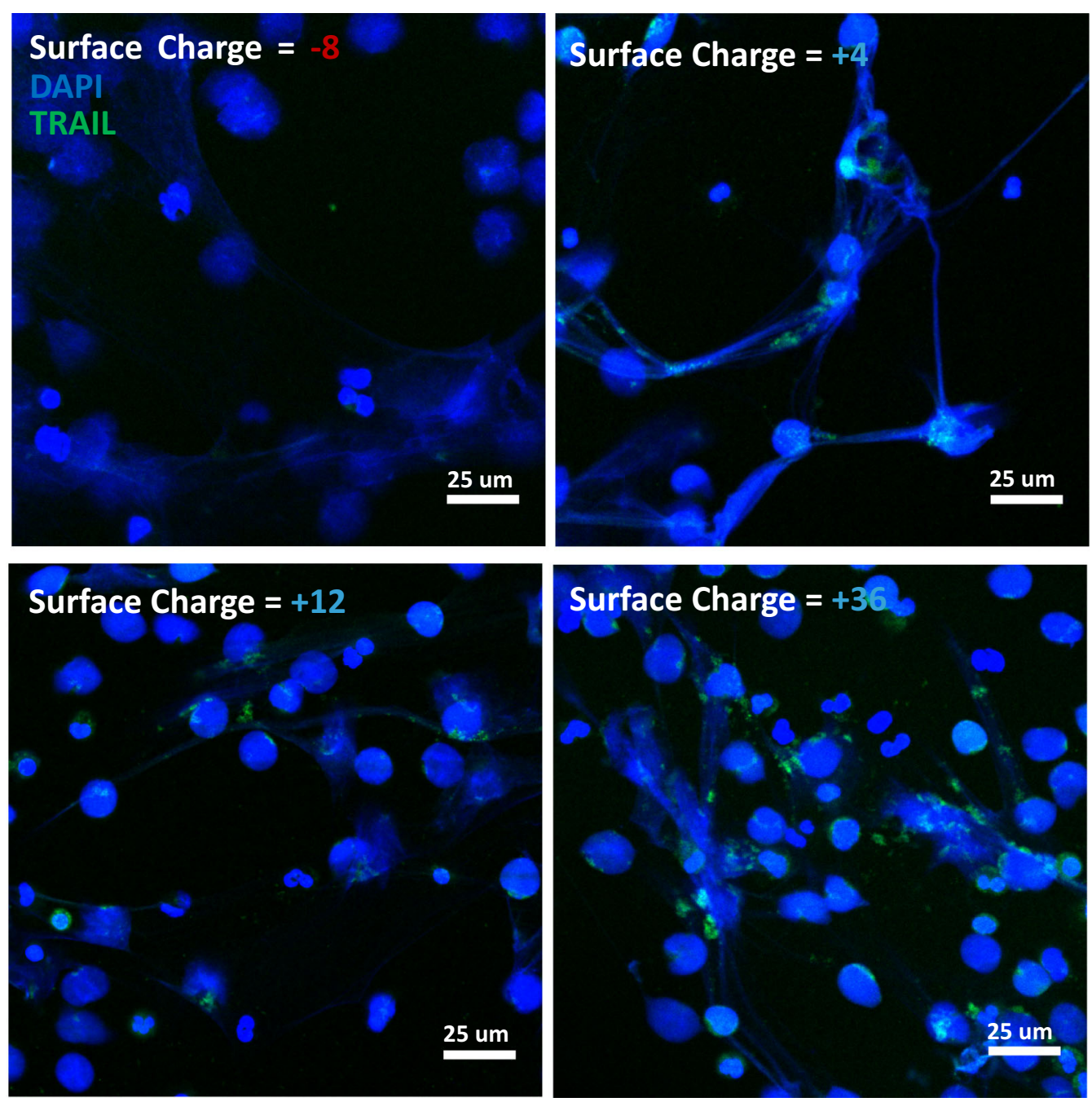

FIGURE 3. The NET-TRAIL complex is mediated by eGFP surface charge. Micrographs have been pseudocolored and overlaid to visually display the degree of overlap between DNA (in blue) and eGFP-TRAIL (in green) for four different net electrostatic charges of the eGFP protein. Note the lack of eGFP-TRAIL colocalized signal in the (-8) negatively charged case.

We examined how the degree of DNA:eGFPTRAIL association depends on the electrostatic surface charge of the supercharged eGFP molecule. It was expected that the net negative charge of the wildtype eGFP protein would need to be manipulated to become positive to enable association with DNA fibers following exteriorization by the NETosis process. As anticipated, the $(-8)$ negatively charged eGFP showed no visible colocalization with DNA nets, however the +4 through +36 eGFP proteins showed visible colocalization with NETs as evidenced by representative fluorescence micrographs (Fig. 3). When an analysis was performed to yield the scalar Pearson Correlation Coefficient (PCC), we observed PCC to be a monotonically increasing function of increased positive charge of the eGFP molecule, indicative of an increasing degree of pixel correlation (Fig. 4). While the most supercharged version of the eGFP protein
$(+36)$ would seem to be the optimal formulation for NETosis delivery based on this PCC analysis, it exhibited significant precipitation and aggregation and was not tested further in cellular activity assays.

Prior to testing the cytotoxic activity of transfected PLB-985 cells, we performed tests to verify that they retain certain neutrophil-like phenotypes. Two of the earliest, molecular indicators of neutrophil activation via the peptide fMLP or inflammatory cytokines are (i) regulation of Mac-1 (CD11b) into an active conformation, and (ii) enzymatic cleavage of L-selectin (CD62L) from the cell surface. ${ }^{10,13}$ Both of these responses occur before shape change and other motility-associated functions, and can be easily assayed via flow cytometry. Figure 5 shows a rightward shift in staining of active CD11b marker using the conformation-specific monoclonal antibody CBRM1/5, as well as a clear leftward shift in surface L-selectin expression 

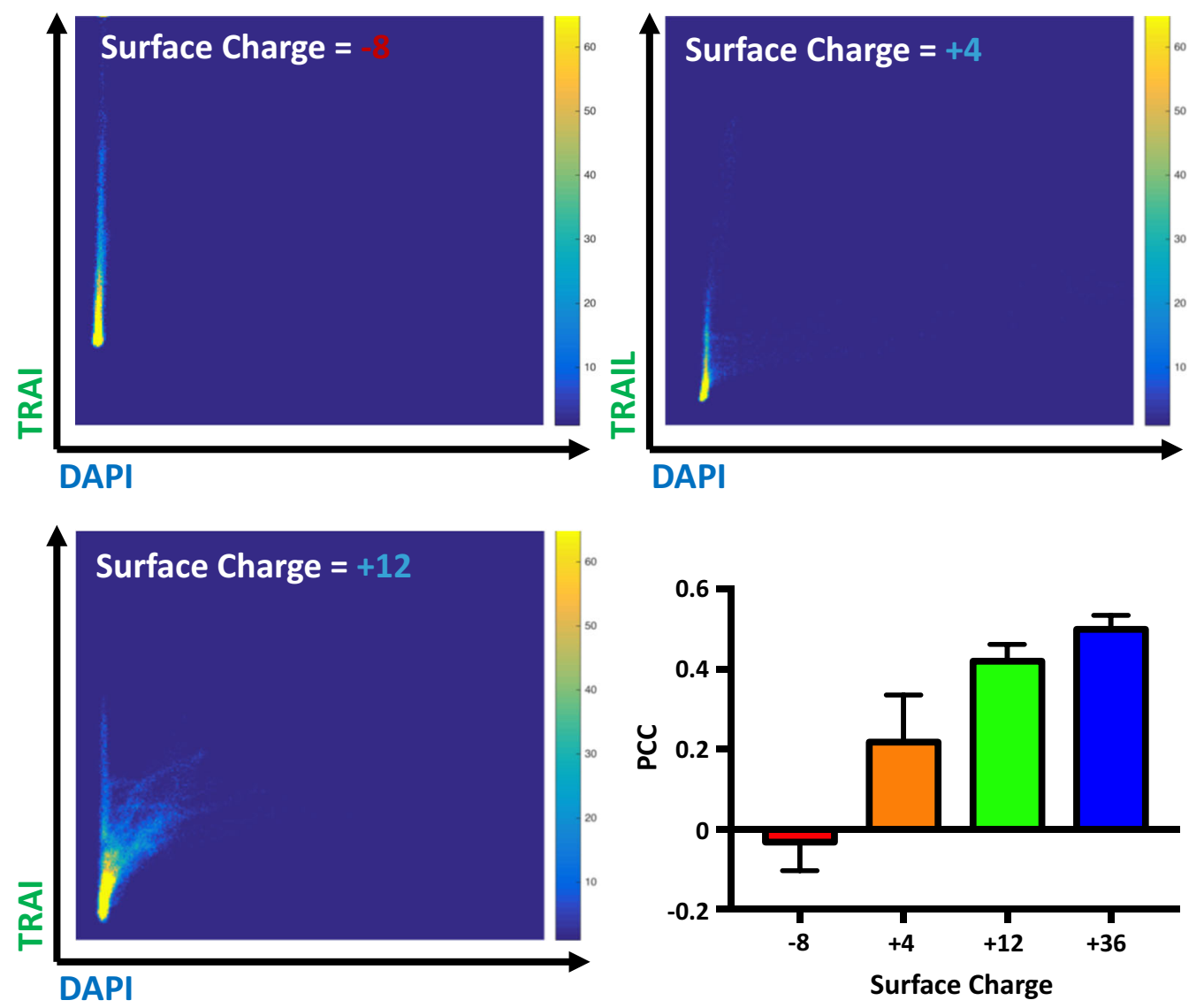

FIGURE 4. The degree of TRAIL/DAPI(DNA) colocalization is a function of the net surface charge of the eGFP molecule. A Pearson correlation coefficient (PCC) analysis was performed on the fluorescence images, shown in representative cases in Fig. 3, and plotted as a function of the eGFP surface charge.

following activation via $10 \mathrm{nM}$ of fMLP. As a later readout of activated neutrophil function, Fig. 6 shows a highly significant fraction of migrating PLB-985 cells following activation via $10 \mathrm{nM}$ of fMLP, and the percentage of cells migrating through a transwell migration assay was similar between the two groups of cells with and without eGFP-TRAIL transfection. From these data we may conclude that these normal neutrophilic functions of acute inflammation may proceed unimpeded while carrying the NETosis-based delivery vehicle.

PLB-985 derived neutrophils expressing NETs decorated with eGFP-TRAIL were co-cultured with multiple human tumor cell lines and showed significant apoptosis-inducing potential. In COLO-205, after $16 \mathrm{~h}$ of co-culture, 7 and $12 \%$ of the tumor cells underwent early and late apoptosis, respectively, while over $55 \%$ of the population were classified as necrotic (Fig. 7a). In the presence of eGFP-decorated NETs, we observed a $>60 \%$ kill rate of cancer cells in the three cell lines SW620, COLO 205 and MDA-MB-231 (Fig. 7b).
SEM images clearly showed significant NETosis when neutrophils were co-cultured with tumor cells. However, only when NETs were decorated with eGFPTRAIL did the tumor cells undergo apoptosis as evident by membrane blebbing (Fig. 7c).

\section{CONCLUSIONS}

The constant presence of neutrophils near tumor cells in all stages of cancer development and in conjunction with the unique ability of neutrophils to undergo NETosis in the presence of cancer cells, make neutrophils a promising candidate for the delivery of cancer therapeutics. Here we introduced a novel form of cancer therapy by leveraging these unique aspects of neutrophils by re-engineering the cell to express a chimeric eGFP-TRAIL protein. This form of treatment exemplifies the potential of employing neutrophils as a drug delivery vehicle that, until now, has been largely unexplored. 

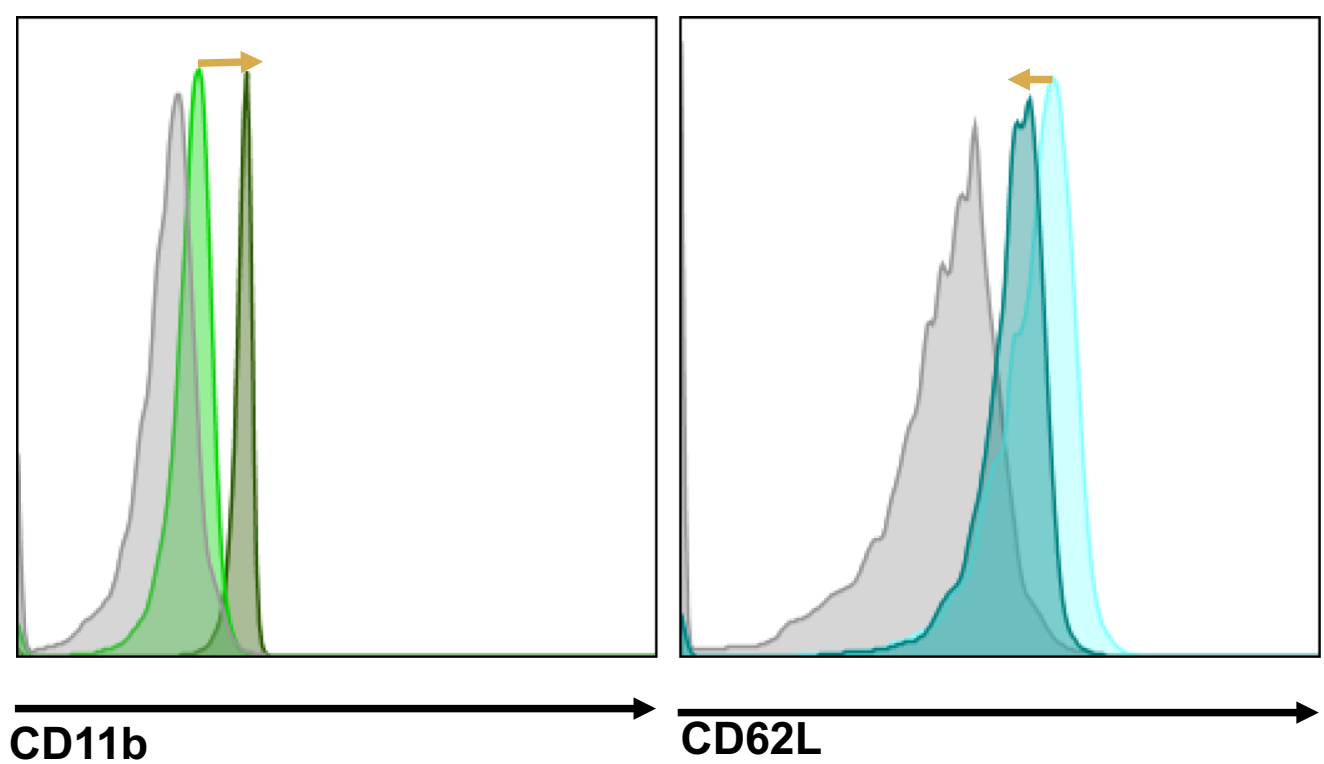

\section{Pre-Treatment}

Post-Treatment

\section{Pre-Treatment \\ Post-Treatment}

FIGURE 5. Transfected PLB-985 cells retain neutrophilic traits after differentiation. Flow cytometric analysis shows that fMLP stimulation of the PLP-985 model neutrophils exhibit the correct molecular traits of early neutrophil activation, including an upregulation of active-conformation CD11b (left graph) and a downregulation of surface CD62L (right graph). Gray curve is isotype control.

\section{METHODS}

\section{Cell Culture and Differentiation}

The acute myeloid leukemia PLB-985 cell line (derivative of HL-60) stably expressing Cas9 protein was a generous gift from the Collins Lab (University of California, Davis). The cells were cultured in RPMI 1640 media supplemented with $2 \mathrm{mM}$ L-glutamine, $25 \mathrm{mM}$ HEPES, $10 \%(\mathrm{v} / \mathrm{v})$ FBS, and $2 \mu \mathrm{g} / \mathrm{mL}$ Blastincidin at $37{ }^{\circ} \mathrm{C}$ and $5 \% \mathrm{CO}_{2}$. Cultured cells were regularly tested for mycoplasma using the Universal Mycoplasma Detection Kit (ATCC 30-1012K).
Neutrophil differentiation of logarithmically growing PLB-985 cells was induced by reduction of FBS to $5 \%$ and supplementation of $0.5 \%$ (vol $/ \mathrm{vol}$ ) DMF. After 3 days, an equivalent of the initial volume of differentiating medium was added and the differentiation continued until day 7 .

\section{Plasma Cell Free DNA Isolation and Quantification}

Plasma was isolated from whole blood of cancer patients and healthy donors by centrifugation, collected after informed consent. To $1 \mathrm{~mL}$ of plasma, 

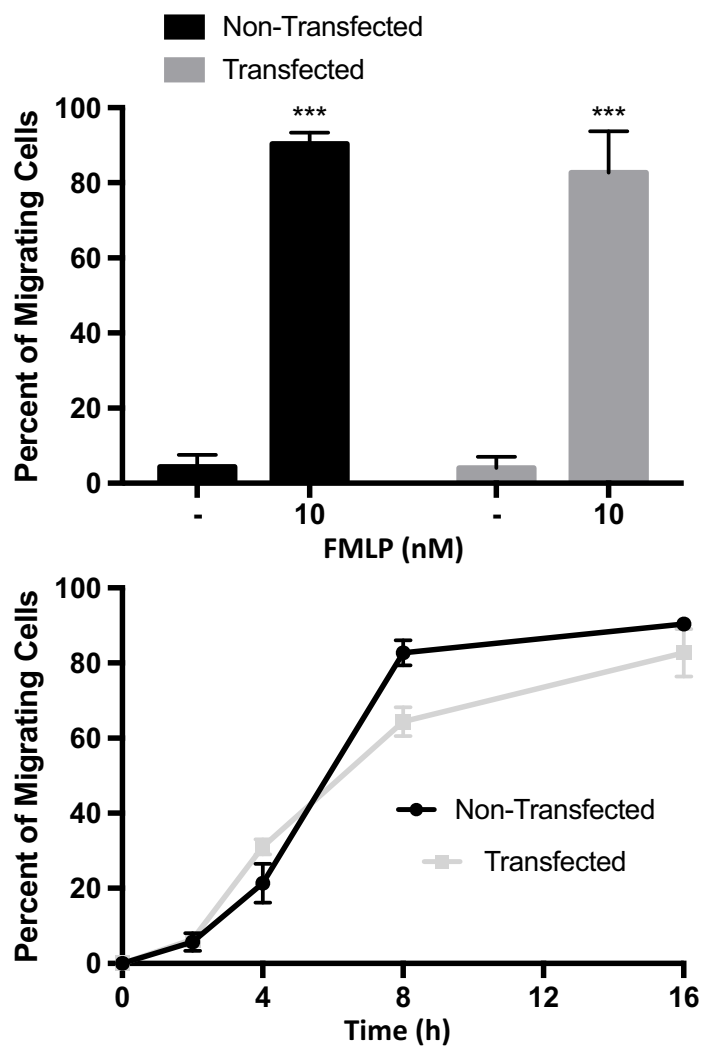

FIGURE 6. Neutrophil-like characteristics are retained after fMLP treatment. eGFP-TRAIL transfected cells showed a similar percentage of migrating cells in the presence of $10 \mathrm{nM}$ of fMLP chemoattractant in a Boyden chamber assay.

$100 \mu \mathrm{L}$ of Tris- $\mathrm{HCl}(\mathrm{pH}$ 8.0) buffer containing $250 \mathrm{mM}$ EDTA, $750 \mathrm{mM} \mathrm{NaCl}, 100 \mathrm{~g} / \mathrm{L}$ of sodium dodecyl sulfate and $20 \mathrm{mg} / \mathrm{mL}$ of proteinase $\mathrm{K}$ was added and incubated for $2 \mathrm{~h}$ at $56^{\circ} \mathrm{C}$. Next, proteins were precipitated with $200 \mu \mathrm{L}$ of $6 \mathrm{M} \mathrm{NaCl}$ solution. The cell-free DNA (cfDNA) was extracted from the supernatant using phenol-chloroform-isoamyl alcohol (25:24:1) followed by ethanol precipitation. DNA concentration was quantified using a Nano-Drop instrument.
Vector Design

pAAVS1-eGFP-TRAIL was constructed starting with the plasmid template of pMK232 (Addgene 72834) containing the AAVS1 homology arms. The eGFP-TRAIL sequence was inserted downstream of the CMV promoter. pX330-U6-AAVS1 was constructed using the plasmid template of pX330 (Addgene 42230). The sgRNA oligonucleotides targeting the AAVS1 region were inserted into the plasmid downstream of the U6 promoter using restriction enzymes AgeI and EcoRI.

Supercharged eGFP-TRAIL DNA sequences were constructed by using site-directed mutagenesis (Agilent) PCR. Indicated amino acids of the wildtype eGFP sequence were replaced with neutral or positively charged amino acids. Protein surface charge was calculated by summing up the total charge of the eGFP sequence based on the predicted charge of the amino acids in the sequence. All plasmid constructs were verified by sequencing.

\section{CRISPR/CAS9 Knock-In of eGFP-TRAIL}

$2 \times 10^{6}$ PLB-985 cells were nucleofected (AMAXA Cell Nucleofector Kit V and Amaxa Nucleofactor II, program C-023) With $20 \mu \mathrm{g}$ of pAAVS1-eGFPTRAIL and $20 \mu \mathrm{g}$ of pX330-U6-AAVS1. Immediately following nucleofection, $500 \mu \mathrm{L}$ of medium was added to the cuvette and the cells incubated at room temperature for $10 \mathrm{~min}$. The cells were then cultured for 48 h. After 48 h, GFP-expressing cells were selected with culture medium supplemented with $2 \mu \mathrm{g} / \mathrm{mL}$ Puromycin.

\section{Chemical and Antibodies}

TACS ${ }^{\circledR}$ Annexin V Kit (Gaithersburg, MD, USA) was used for assaying cell apoptosis. Reagents for SEM were obtained from Electron Microscopy Sciences (Hatfield, PA, USA): glutaraldehyde, osmium tetroxide and uranyl acetate. Antibodies for human 

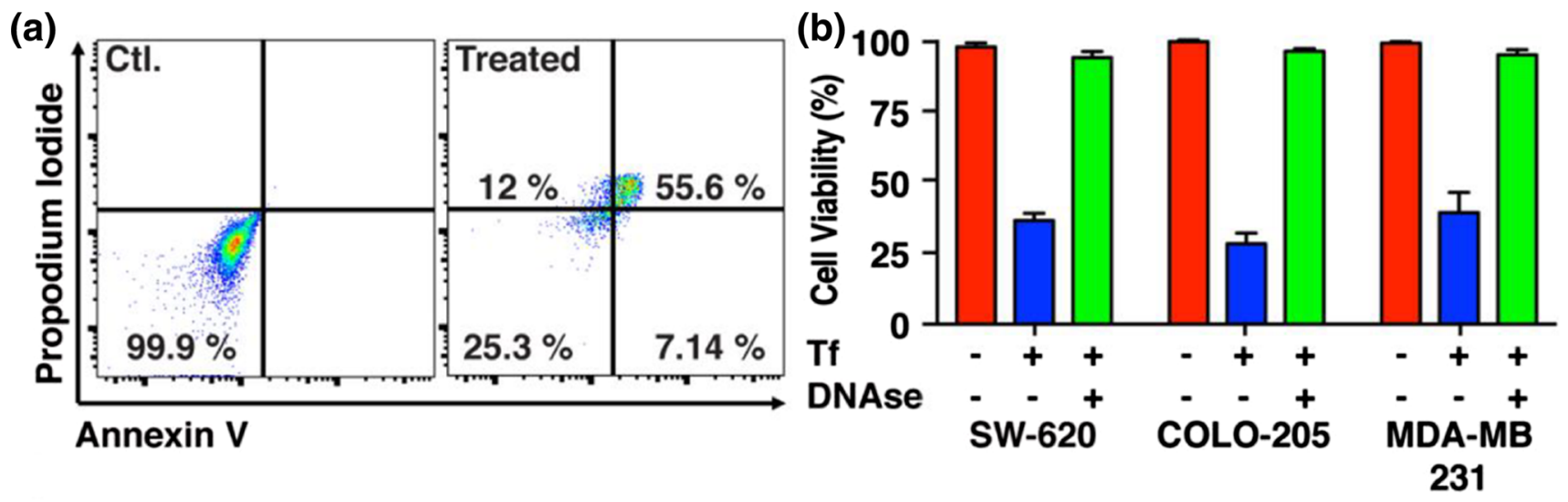

(c)
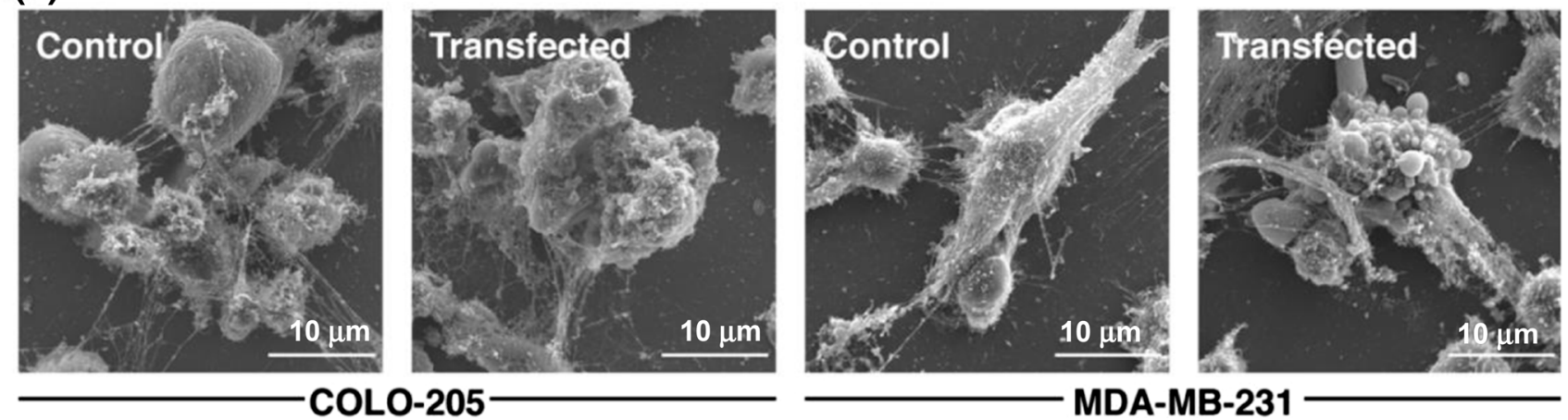

MDA-MB-231

FIGURE 7. Supercharged eGFP-TRAIL expressing neutrophils trap and destroy tumor cells during NETosis. (a) Representative flow cytometry result of COLO-205 cells co-cultured with eGFP-TRAIL expressing neutrophils for $24 \mathrm{~h}$ and stained for apoptosis and necrosis using Annexin V and propodium iodide, respectively. (b) Cell viability quantification of indicated cancer cell lines cocultured with wildtype neutrophils or cells positive for eGFP-TRAIL (Tf). (c) Immuno-gold SEM images of indicated cancer cells cocultured with neutrophils.

TRAIL were purchased from Biolegend (San Diego, CA, USA). Primary antibodies for human TRAIL and $\beta$-actin were obtained from PeproTech (Rocky Hill, NJ, USA) and Santa Cruz Biotech (Santa Cruz, CA, USA). HRP-conjugated anti-mouse and anti-rabbit antibodies were obtained from Santa Cruz Biotech. IL8 human Elisa Kits were purchased from ThermoFisher.

\section{CONFLICT OF INTEREST}

The authors T.C. and M.R.K. have no conflicts of interest to disclose.

\section{ETHICS APPROVAL}

Blood samples were collected from human participants after informed consent, following a protocol approved by the Institutional Review Board of Vanderbilt University. No animal studies were performed in this study.

\section{OPEN ACCESS}

This article is licensed under a Creative Commons Attribution 4.0 International License, which permits use, sharing, adaptation, distribution and reproduction in any medium or format, as long as you give appropriate credit to the original author(s) and the source, provide a link to the Creative Commons licence, and indicate if changes were made. The images or other third party material in this article are included in the article's Creative Commons licence, unless indicated otherwise in a credit line to the material. If material is not included in the article's Creative Commons licence and your intended use is not permitted by statutory regulation or exceeds the permitted use, you will need to obtain permission directly from the copyright holder. To view a copy of this licence, visit http://crea tivecommons.org/licenses/by/4.0/.

\section{REFERENCES}

\footnotetext{
${ }^{1}$ Albelda, S. M., et al. Polarization of tumor-associated neutrophil phenotype by TGF- $\beta$ : "N1" versus "N2" TAN. Cancer Cell 16:183-194, 2009.
} 
${ }^{2}$ Atkins, M., et al. Arginase I-producing myeloid-derived suppressor cells in renal cell carcinoma are a subpopulation of activated granulocytes. Cancer Res. 69:1553-1560, 2009.

${ }^{3}$ Brinkmann, V., et al. Neutrophil extracellular traps kill bacteria. Science. 303:1532-1535, 2004.

${ }^{4}$ De Larco, J. E., B. R. K. Wuertz, L. T. Furcht, and J. E. D. Larco. The potential role of neutrophils in promoting the metastatic phenotype of tumors releasing interleukin- 8 the potential role of neutrophils in promoting the metastatic phenotype of tumors releasing interleukin-8. Clin. Cancer Res. 10:4895-4900, 2004.

${ }^{5}$ Demers, M., et al. Cancers predispose neutrophils to release extracellular DNA traps that contribute to cancerassociated thrombosis. Proc. Natl. Acad. Sci. 109:1307613081, 2012.

${ }^{6}$ Giannias, B., et al. Neutrophil extracellular traps sequester circulating tumor cells and promote metastasis. J. Clin. Invest. 123:3446-3458, 2013.

${ }^{7}$ Godschalk, R. W. L., et al. Genotoxic effects of neutrophils and hypochlorous acid. Mutagenesis 25:149-154, 2009.

${ }^{8}$ Gregory, A. D., and A. M. Houghton. Tumor-associated neutrophils: new targets for cancer therapy. Cancer Research 71:2411-2416, 2011.

${ }^{9}$ Jurk, D., et al. $\mathrm{NF} \kappa \mathrm{B} 1$ is a suppressor of neutrophil-driven hepatocellular carcinoma. Nat. Commun. 6:6818, 2015.

${ }^{10}$ Lee, D., J. B. Schultz, P. A. Knauf, and M. R. King. Mechanical shedding of L-selectin from the neutrophil surface during rolling on sialyl Lewis x under flow. J. Biol. Chem. 282:4812-4820, 2007.

${ }^{11} \mathrm{Li}$, J., C. C. Sharkey, B. Wun, J. L. Liesveld, and M. R. King. Genetic engineering of platelets to neutralize circulating tumor cells. J. Control. Release 228:38-47, 2016.

${ }^{12}$ Lievin-Le Moal, V., et al. Afa/Dr diffusely adhering Escherichia coli strain C1845 induces neutrophil extracellular traps that kill bacteria and damage human enterocyte-like cells. Infect. Immun. 80:1891-1899, 2012.
${ }^{13}$ Mitchell, M. J., and M. R. King. Shear-induced resistance to neutrophil activation via the formyl peptide receptor. Biophys. J. 102:1804-1814, 2012.

${ }^{14}$ Mitchell, M. J., E. Wayne, K. Rana, C. B. Schaffer, and M. R. King. TRAIL-coated leukocytes that kill cancer cells in the circulation. Proc. Natl. Acad. Sci. 111:930-935, 2014.

${ }^{15}$ Nozawa, H., C. Chiu, and D. Hanahan. Infiltrating neutrophils mediate the initial angiogenic switch in a mouse model of multistage carcinogenesis. Proc. Natl. Acad. Sci. 103:12493-12498, 2006.

${ }^{16}$ Papayannopoulos, V. Neutrophil extracellular traps in immunity and disease. Nat. Rev. Immunol. 18:134-147, 2018.

${ }^{17}$ Pedruzzi, E., M. Fay, C. Elbim, M. Gaudry, and M.-A. Gougerot-Pocidalo. Differentiation of PLB-985 myeloid cells into mature neutrophils, shown by degranulation of terminally differentiated compartments in response to $\mathrm{N}$ formyl peptide and priming of superoxide anion production by granulocyte-macrophage colony-stimulating fact. Br. J. Haematol. 117:719-726, 2002.

${ }^{18}$ Szczerba, B. M., et al. Neutrophils escort circulating tumour cells to enable cell cycle progression. Nature 566:553$557,2019$.

${ }^{19}$ Thacker, S. G., et al. A distinct subset of proinflammatory neutrophils isolated from patients with systemic lupus erythematosus induces vascular damage and synthesizes type I IFNs. J. Immunol. 184:3284-3297, 2010.

${ }^{20}$ Wculek, S. K., and I. Malanchi. Neutrophils support lung colonization of metastasis-initiating breast cancer cells. Nature 528:413-417, 2015.

Publisher's Note Springer Nature remains neutral with regard to jurisdictional claims in published maps and institutional affiliations. 